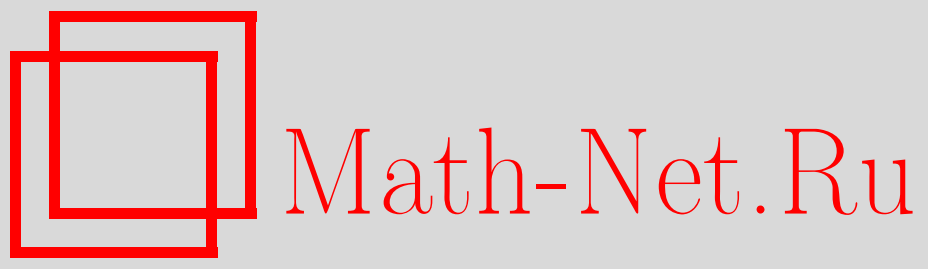

Ю. М. Мешкова, Т. А. Суслина, Усреднение решений начально-краевых задач для параболических систем, Функи. анализ и его прил., 2015, том 49, выпуск 1, 88-93

DOI: https://doi.org/10.4213/faa3177

Использование Общероссийского математического портала MathNet.Ru подразумевает, что вы прочитали и согласны с пользовательским соглашением

http://www. mathnet.ru/rus/agreement

Параметры загрузки:

IP : 54.237 .59 .107

26 апреля 2023 г., 12:04:07

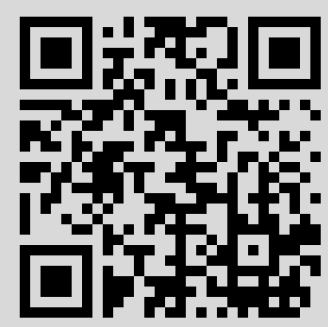


УДК 517.956.4

\title{
Усреднение решений начально-краевых задач для параболических систем*
}

\author{
(c) 2015. Ю. М. Мешкова, Т. А. СуСлина
}

В работе изучается усреднение (гомогенизация) решений параболических систем с периодическими коэффициентами. Этой задаче много внимания уделяется в книгах по теории усреднений (см., например, [1]-[3]). Операторнъе оценки погрешности для таких задач в $\mathbb{R}^{d}$ изучались в [4]-[10]. В настоящей работе представлены результаты по усреднению решений начально-краевых задач для параболических систем в ограниченной области. При этом мы опираемся на недавние результаты по усреднению эллиптических краевых задач ([11]-[14]).

1. Класс операторов. Пусть $\Gamma$ - решетка в $\mathbb{R}^{d}$ и $\Omega$ - элементарная ячейка этой решетки. Для всякой Г-периодической функции $\varphi(\mathbf{x})$ используем обозначение $\varphi^{\varepsilon}(\mathbf{x})=\varphi(\mathbf{x} / \varepsilon), \varepsilon>0$. Пусть $g(\mathbf{x})$ есть $\Gamma$-периодическая эрмитова матрицафункция размера $m \times m$. Предполагается, что $g(\mathbf{x})$ положительно определена и $g, g^{-1} \in L_{\infty}$. Пусть $b(\mathbf{D})$ есть $(m \times n)$-матричный дифференциальный оператор вида $b(\mathbf{D})=\sum_{l=1}^{d} b_{l} D_{l}$, где $b_{l}$ - постоянные матрицы. Оператору $b(\mathbf{D})$ отвечает символ $b(\boldsymbol{\xi})=\sum_{l=1}^{d} b_{l} \xi_{l}, \boldsymbol{\xi} \in \mathbb{R}^{d}$. Предполагается, что $m \geqslant n$ и

$$
\operatorname{rank} b(\boldsymbol{\xi})=n, \quad 0 \neq \boldsymbol{\xi} \in \mathbb{R}^{d} .
$$

Это равносильно выполнению неравенств $\alpha_{0} \mathbf{1}_{n} \leqslant b(\boldsymbol{\theta})^{*} b(\boldsymbol{\theta}) \leqslant \alpha_{1} \mathbf{1}_{n}$ при $\boldsymbol{\theta} \in \mathbb{S}^{d-1}$ с некоторыми $\alpha_{0}, \alpha_{1}>0$.

Пусть $\mathscr{O} \subset \mathbb{R}^{d}-$ ограниченная область с границей класса $C^{1,1}$. В $L_{2}\left(\mathscr{O} ; \mathbb{C}^{n}\right)$ рассмотрим оператор $A_{D, \varepsilon}$, формально заданный дифференциальным выражением $b(\mathbf{D})^{*} g^{\varepsilon}(\mathbf{x}) b(\mathbf{D})$ с краевым условием Дирихле. Строго говоря, $A_{D, \varepsilon}-$ самосопряженный оператор, порожденный замкнутой и положительно определенной квадратичной формой $\left(g^{\varepsilon} b(\mathbf{D}) \mathbf{u}, b(\mathbf{D}) \mathbf{u}\right)_{L_{2}(\mathscr{O})}, \mathbf{u} \in H_{0}^{1}\left(\mathscr{O} ; \mathbb{C}^{n}\right)$. Здесь $H_{0}^{1}\left(\mathscr{O} ; \mathbb{C}^{n}\right)$ - замыкание множества $C_{0}^{\infty}\left(\mathscr{O} ; \mathbb{C}^{n}\right)$ по норме пространства $H^{1}\left(\mathscr{O} ; \mathbb{C}^{n}\right)$.

В случае условия Неймана наложим более сильное ограничение, чем (1):

$$
\operatorname{rank} b(\boldsymbol{\xi})=n, \quad 0 \neq \boldsymbol{\xi} \in \mathbb{C}^{d} .
$$

Как показано в [15], условие (2) необходимо и достаточно для коэрцитивности формы $\|b(\mathbf{D}) \mathbf{u}\|_{L_{2}(\mathscr{O})}^{2}$ : существуют постоянные $c_{1}>0$ и $c_{2} \geqslant 0$, такие, что

$$
\|b(\mathbf{D}) \mathbf{u}\|_{L_{2}(\mathscr{O})}^{2}+c_{2}\|\mathbf{u}\|_{L_{2}(\mathscr{O})}^{2} \geqslant c_{1}\|\mathbf{D u}\|_{L_{2}(\mathscr{O})}^{2}, \quad \mathbf{u} \in H^{1}\left(\mathscr{O} ; \mathbb{C}^{n}\right)
$$

Тогда форма $\left(g^{\varepsilon} b(\mathbf{D}) \mathbf{u}, b(\mathbf{D}) \mathbf{u}\right)_{L_{2}(\mathscr{O})}, \mathbf{u} \in H^{1}\left(\mathscr{O} ; \mathbb{C}^{n}\right)$, замкнута и неотрицательна. Порожденный ею оператор в $L_{2}\left(\mathscr{O} ; \mathbb{C}^{n}\right)$ обозначим через $A_{N, \varepsilon}$. Формально он задан выражением $b(\mathbf{D})^{*} g^{\varepsilon}(\mathbf{x}) b(\mathbf{D})$ с краевым условием Неймана $\left.\partial_{\boldsymbol{\nu}}^{\varepsilon} \mathbf{u}\right|_{\partial \mathscr{O}}=0$ (здесь $\partial_{\nu}^{\varepsilon} \mathbf{u}$ означает соответствующую конормальную производную).

* Работа выполнена при поддержке РФФИ (проект 14-01-00760). Первый автор поддержан Лабораторией им. П. Л. Чебышева СПбГУ, грант Правительства РФ, дог. 11.G34.31.0026, и OAO «Газпром нефть». 
Чтобы избежать дублирования формулировок, используем значок $b=D, N$. Ниже, если $b=D$, предполагается выполненным условие $(1)$, в в случае $b=N$ - условие (2).

Нас интересует поведение операторной экспоненты $e^{-A_{b, \varepsilon} t}, t>0$, при малом $\varepsilon$. Выясняется, что при фиксированном $t>0$ и $\varepsilon \rightarrow 0$ оператор $e^{-A_{b, \varepsilon} t}$ сходится по операторной норме в $L_{2}$ к экспоненте $e^{-A_{b}^{0} t}$ от эффективного оператора.

2. Эффективный оператор. Пусть $(n \times m)$-матрица-функция $\Lambda(\mathbf{x})$ является (слабым) Г-периодическим решением задачи

$$
b(\mathbf{D})^{*} g(\mathbf{x})\left(b(\mathbf{D}) \Lambda(\mathbf{x})+\mathbf{1}_{m}\right)=0, \quad \int_{\Omega} \Lambda(\mathbf{x}) d \mathbf{x}=0 .
$$

(Отметим, что $\Lambda$ принадлежит $H_{\mathrm{per}}^{1}(\Omega)$, но в общем случае может не быть ограниченной.) Эффективная матрица $g^{0}$ размера $m \times m$ определена выражением

$$
g^{0}=|\Omega|^{-1} \int_{\Omega} \tilde{g}(\mathbf{x}) d \mathbf{x}, \quad \text { где } \tilde{g}(\mathbf{x}):=g(\mathbf{x})\left(b(\mathbf{D}) \Lambda(\mathbf{x})+\mathbf{1}_{m}\right) .
$$

Матрица $g^{0}$ положительна. Эффективный оператор $A_{D}^{0}$ задается выражением $b(\mathbf{D})^{*} g^{0} b(\mathbf{D})$ на области определения $H^{2}\left(\mathscr{O} ; \mathbb{C}^{n}\right) \cap H_{0}^{1}\left(\mathscr{O} ; \mathbb{C}^{n}\right)$. Эфбфективныи оператор $A_{N}^{0}$ задается выражением $b(\mathbf{D})^{*} g^{0} b(\mathbf{D})$ на функциях из $H^{2}\left(\mathscr{O} ; \mathbb{C}^{n}\right)$, удовлетворяющих условию $\left.\partial_{\nu}^{0} \mathbf{u}\right|_{\partial \mathscr{O}}=0$ (здесь $\partial_{\nu}^{0} \mathbf{u}$ означает конормальную производную, отвечающую оператору $\left.b(\mathbf{D})^{*} g^{0} b(\mathbf{D})\right)$.

3. Основные результаты. Операторы $A_{D, \varepsilon}$ и $A_{D}^{0}$ положительно определены. Число $c_{D}^{\circ}=\alpha_{0}\left\|g^{-1}\right\|_{L_{\infty}^{-1}}(\operatorname{diam} \mathscr{O})^{-2}$ является их общей нижней гранью. Положим $c_{D}=c_{D}^{\circ} / 2$.

Для операторов $A_{N, \varepsilon}$ и $A_{N}^{0}$ точка $\lambda=0$ является собственным значением, причем собственные подпространства совпадают: $\operatorname{Ker} A_{N, \varepsilon}=\operatorname{Ker} A_{N}^{0}=Z$, где $Z:=\operatorname{Ker} b(\mathbf{D})$ - конечномерное подпространство в $H^{1}\left(\mathscr{O} ; \mathbb{C}^{n}\right)$. Положим $H_{\perp}^{1}\left(\mathscr{O} ; \mathbb{C}^{n}\right)=\left\{\mathbf{u} \in H^{1}\left(\mathscr{O} ; \mathbb{C}^{n}\right):(\mathbf{u}, \mathbf{z})_{L_{2}(\mathscr{O})}=0\right.$ для любого $\left.\mathbf{z} \in Z\right\}$. Через $\mathscr{P}_{Z}$ обозначим ортопроектор в $L_{2}\left(\mathscr{O} ; \mathbb{C}^{n}\right)$ на $Z$. Отметим очевидное тождество $e^{-A_{N, \varepsilon} t}-e^{-A_{N}^{0} t}=\left(e^{-A_{N, \varepsilon} t}-e^{-A_{N}^{0} t}\right)\left(I-\mathscr{P}_{Z}\right)$. Согласно [13], существует постоянная $c_{3}$, такая, что

$$
\|\mathbf{u}\|_{H^{1}(\mathscr{O})}^{2} \leqslant c_{3}\|b(\mathbf{D}) \mathbf{u}\|_{L_{2}(\mathscr{O})}^{2}, \quad \mathbf{u} \in H_{\perp}^{1}\left(\mathscr{O} ; \mathbb{C}^{n}\right)
$$

Число $c_{N}^{\circ}=\left\|g^{-1}\right\|_{L_{\infty}}^{-1} c_{3}^{-1}$ заведомо не превосходит $\mu_{2}^{\varepsilon}$ и $\mu_{2}^{0}$, где $\mu_{2}^{\varepsilon}$ (соответственно $\left.\mu_{2}^{0}\right)$ - первое ненулевое собственное значение оператора $A_{N, \varepsilon}$ (соответственно $\left.A_{N}^{0}\right)$. Положим $c_{N}=c_{N}^{\circ} / 2$.

Теорема 1. Найдется число $\varepsilon_{0} \in(0,1]$, зависящее лишь от области $\mathscr{O} u$ решетки $\Gamma$, такое, что при $0<\varepsilon \leqslant \varepsilon_{0}$ справедлива оценка

$$
\left\|e^{-A_{\mathrm{b}, \varepsilon} t}-e^{-A_{\mathrm{b}}^{0} t}\right\|_{L_{2}(\mathscr{O}) \rightarrow L_{2}(\mathscr{O})} \leqslant C_{1}^{b} e^{-c_{\mathrm{b}} t} \varepsilon\left(t+\varepsilon^{2}\right)^{-1 / 2}, \quad t \geqslant 0, b=D, N .
$$

При аппроксимации оператора $e^{-A_{b, \varepsilon} t}$ по $\left(L_{2} \rightarrow H^{1}\right)$-норме необходимо учитывать корректор. Фиксируем линейный непрерывный оператор продолжения $P_{\mathscr{O}}: H^{2}\left(\mathscr{O} ; \mathbb{C}^{n}\right) \rightarrow H^{2}\left(\mathbb{R}^{d} ; \mathbb{C}^{n}\right)$. Требуется, чтобы $P_{\mathscr{O}}$ одновременно был непрерывным оператором из $L_{2}\left(\mathscr{O} ; \mathbb{C}^{n}\right)$ в $L_{2}\left(\mathbb{R}^{d} ; \mathbb{C}^{n}\right)$ и из $H^{1}\left(\mathscr{O} ; \mathbb{C}^{n}\right)$ в $H^{1}\left(\mathbb{R}^{d} ; \mathbb{C}^{n}\right)$. 
Такой оператор существует (см., например, [16]). Через $R_{\mathscr{O}}$ обозначим оператор сужения функций в $\mathbb{R}^{d}$ на область $\mathscr{O}$. Корректор в случае условия Дирихле определяется выражением $K_{D}(t ; \varepsilon):=R_{\mathscr{O}} \Lambda^{\varepsilon} S_{\varepsilon} b(\mathbf{D}) P_{\mathscr{O}} e^{-A_{D}^{0} t}$, а в случае условия Неймана - выражением $K_{N}(t ; \varepsilon):=R_{\mathscr{O}} \Lambda^{\varepsilon} S_{\varepsilon} b(\mathbf{D}) P_{\mathscr{O}} e^{-A_{N}^{0} t}\left(I-\mathscr{P}_{Z}\right)$. В общем случае корректор $K_{b}(t ; \varepsilon)$ содержит вспомогательный оператор $S_{\varepsilon}$, определенный равенством $\left(S_{\varepsilon} \mathbf{u}\right)(\mathbf{x})=|\Omega|^{-1} \int_{\Omega} \mathbf{u}(\mathbf{x}-\varepsilon \mathbf{z}) d \mathbf{z}, \mathbf{u} \in L_{2}\left(\mathbb{R}^{d} ; \mathbb{C}^{m}\right)$, и называемый сглаживающим оператором по Стеклову. Используя свойства оператора $S_{\varepsilon}$ и включение $\Lambda \in H_{\text {per }}^{1}(\Omega)$, нетрудно убедиться, что оператор $K_{b}(t ; \varepsilon)$ непрерывно переводит $L_{2}\left(\mathscr{O} ; \mathbb{C}^{n}\right)$ в $H^{1}\left(\mathscr{O} ; \mathbb{C}^{n}\right)$. При дополнительном условии $\Lambda \in L_{\infty}$ функция $\Lambda$ оказывается мультипликатором в $H^{1}(\mathscr{O})$ (см. [11]), и тогда можно использовать обычный корректор $K_{b}^{0}(t ; \varepsilon):=\Lambda^{\varepsilon} b(\mathbf{D}) e^{-A_{b}^{0} t}$, который в этом случае является ограниченным оператором из $L_{2}\left(\mathscr{O} ; \mathbb{C}^{n}\right)$ в $H^{1}\left(\mathscr{O} ; \mathbb{C}^{n}\right)$. Следующая теорема дает $\left(L_{2} \rightarrow H^{1}\right)$-аппроксимацию экспоненты $e^{-A_{b, \varepsilon} t}$, а также $\left(L_{2} \rightarrow L_{2}\right)$-аппроксимацию оператора $g^{\varepsilon} b(\mathbf{D}) e^{-A_{b, \varepsilon} t}$. Использованы обозначения $G_{D}(t ; \varepsilon):=\tilde{g}^{\varepsilon} S_{\varepsilon} b(\mathbf{D}) P_{\mathscr{O}} e^{-A_{D}^{0} t}, G_{N}(t ; \varepsilon):=\tilde{g}^{\varepsilon} S_{\varepsilon} b(\mathbf{D}) P_{\mathscr{O}} e^{-A_{N}^{0} t}\left(I-\mathscr{P}_{Z}\right)$.

Теорема 2. Найдется число $\varepsilon_{0} \in(0,1]$, зависящее лишь от области $\mathscr{O} u$ решетки $\Gamma$, такое, что при $b=D, N$ имеют место следующие утверждения.

$1^{\circ}$. При $0<\varepsilon \leqslant \varepsilon_{0}$ u $t>0$ справедливы оченки

$$
\begin{aligned}
\left\|e^{-A_{b, \varepsilon} t}-e^{-A_{b}^{0} t}-\varepsilon K_{b}(t ; \varepsilon)\right\|_{L_{2}(\mathscr{O}) \rightarrow H^{1}(\mathscr{O})} & \leqslant C_{2}^{b} e^{-c_{b} t}\left(\varepsilon^{1 / 2} t^{-3 / 4}+\varepsilon t^{-1}\right), \\
\left\|g^{\varepsilon} b(\mathbf{D}) e^{-A_{b, \varepsilon} t}-G_{b}(t ; \varepsilon)\right\|_{L_{2}(\mathscr{O}) \rightarrow L_{2}(\mathscr{O})} & \leqslant C_{3}^{b} e^{-c_{b} t}\left(\varepsilon^{1 / 2} t^{-3 / 4}+\varepsilon t^{-1}\right) .
\end{aligned}
$$

$2^{\circ}$. Пусть $\Lambda \in L_{\infty}$. При $0<\varepsilon \leqslant \varepsilon_{0}$ u $t>0$ справедливы оченки

$$
\begin{aligned}
& \left\|e^{-A_{b, \varepsilon} t}-e^{-A_{b}^{0} t}-\varepsilon K_{b}^{0}(t ; \varepsilon)\right\|_{L_{2}(\mathscr{O}) \rightarrow H^{1}(\mathscr{O})} \leqslant \widetilde{C}_{2}^{b} e^{-c_{b} t}\left(\varepsilon^{1 / 2} t^{-3 / 4}+\varepsilon t^{-1}\right), \\
& \left\|g^{\varepsilon} b(\mathbf{D}) e^{-A_{b, \varepsilon} t}-\tilde{g}^{\varepsilon} b(\mathbf{D}) e^{-A_{b}^{0} t}\right\|_{L_{2}(\mathscr{O}) \rightarrow L_{2}(\mathscr{O})} \leqslant \widetilde{C}_{3}^{b} e^{-c_{b} t}\left(\varepsilon^{1 / 2} t^{-3 / 4}+\varepsilon t^{-1}\right) .
\end{aligned}
$$

Отметим, что $\varepsilon t^{-1} \leqslant \varepsilon^{1 / 2} t^{-3 / 4}$ при $t \geqslant \varepsilon^{2}$. При $t<\varepsilon^{2}$ неравенства (6)-(9) «портятся» и приходится применять достаточно грубые оценки.

Предложение 3. При $t>0 u 0<\varepsilon \leqslant 1$ справедливы оценки

$$
\begin{aligned}
\left\|e^{-A_{b, \varepsilon} t}-e^{-A_{b}^{0} t}\right\|_{L_{2}(\mathscr{O}) \rightarrow H^{1}(\mathscr{O})} \leqslant C_{4}^{b} t^{-1 / 2} e^{-c_{b} t}, & b & =D, N, \\
\left\|g^{\varepsilon} b(\mathbf{D}) e^{-A_{b, \varepsilon} t}\right\|_{L_{2}(\mathscr{O}) \rightarrow L_{2}(\mathscr{O})} \leqslant C_{5}^{b} t^{-1 / 2} e^{-c_{b} t}, & b & =D, N .
\end{aligned}
$$

Замечание 4. (i) Постоянные $C_{j}^{D}$ из неравенств (5)-(7), (10), (11) (с b = D) зависят от $d, m,\|g\|_{L_{\infty}},\left\|g^{-1}\right\|_{L_{\infty}}, \alpha_{0}, \alpha_{1}$, от параметров решетки Г и от области $\mathscr{O}$, а постоянные $\widetilde{C}_{j}^{D}$ из неравенств $(8),(9)($ с $b=D)$ зависят от тех же величин и еще от $\|\Lambda\|_{L_{\infty}}$. (ii) Постоянные $C_{j}^{N}$ из неравенств (5)-(7), (10), (11) (с b $=N$ ) зависят от $d, n, m, \operatorname{dim} Z,\|g\|_{L_{\infty}},\left\|g^{-1}\right\|_{L_{\infty}}, \alpha_{0}, \alpha_{1}$, от параметров решетки $\Gamma$, от области $\mathscr{O}$, а также от постоянных $c_{1}, c_{2}, c_{3}$ из неравенств (3), (4). Постоянные $\widetilde{C}_{j}^{N}$ из неравенств $(8),(9)($ c $b=N)$ зависят от перечисленных величин и еще от $\|\Lambda\|_{L_{\infty}}$. (iii) Пусть $\delta>0$ - произвольное достаточно малое число. Если считать $\varepsilon$ достаточно малым, то в оценках (5)-(11) с $b=D$ можно принять $c_{D}=\lambda_{1}^{0}-\delta$, где $\lambda_{1}^{0}$ - первое собственное значение оператора $A_{D}^{0}$, а в оценках (5)-(11) с $b=N$ можно принять $c_{N}=\mu_{2}^{0}-\delta$. Тогда константы в оценках (5)-(11) будут зависеть еще и от $\delta$. 
Отметим, что порядок $\varepsilon\left(t+\varepsilon^{2}\right)^{-1 / 2}$ оценки (5) получился таким же, как для задачи в $\mathbb{R}^{d}$ (ср. [4], [5]). Разница лишь в множителе $e^{-c_{b} t}$ (для задачи в $\mathbb{R}^{d}$ он отсутствует, поскольку точка нуль является краем непрерывного спектра). Порядок оценки $O(\varepsilon)$ при фиксированном $t$ точный. Что касается оценок из теоремы 2 , то при фиксированном $t \geqslant \varepsilon^{2}$ они имеют порядок $O\left(\varepsilon^{1 / 2}\right)$, в то время как в $\mathbb{R}^{d}$ был порядок $O(\varepsilon)$ (ср. [7]). Это связано с влиянием границы и согласуется с результатами для эллиптических краевых задач ([11]-[14]).

Для доказательства теорем 1, 2 применяются представления операторов $e^{-A_{b, \varepsilon} t}, e^{-A_{b}^{0} t}$ в виде интегралов от соответствующих резольвент по подходящему контуру в комплексной плоскости и результаты работы [14] об аппроксимации резольвенты $\left(A_{b, \varepsilon}-\zeta I\right)^{-1}$.

\section{4. Применение к усреднению решений начально-краевых задач.} Пусть $\mathbf{u}_{b, \varepsilon}(\mathbf{x}, t)$ - обобщенное решение задачи

$$
\begin{cases}\frac{\partial \mathbf{u}_{b, \varepsilon}(\mathbf{x}, t)}{\partial t}=-b(\mathbf{D})^{*} g^{\varepsilon}(\mathbf{x}) b(\mathbf{D}) \mathbf{u}_{b, \varepsilon}(\mathbf{x}, t)+\mathbf{F}(\mathbf{x}, t), & \mathbf{x} \in \mathscr{O}, 0<t<T, \\ \mathbf{u}_{b, \varepsilon}(\mathbf{x}, 0)=\phi(\mathbf{x}), \quad \mathbf{x} \in \mathscr{O} & \\ \left.\mathbf{u}_{D, \varepsilon}(\cdot, t)\right|_{\partial \mathscr{O}}=0 \quad \text { либо }\left.\quad \partial_{\nu}^{\varepsilon} \mathbf{u}_{N, \varepsilon}(\cdot, t)\right|_{\partial \mathscr{O}}=0, \quad 0<t<T,\end{cases}
$$

где $\phi \in L_{2}\left(\mathscr{O} ; \mathbb{C}^{n}\right)$ и $\mathbf{F} \in \mathscr{H}_{p}(T):=L_{p}\left((0, T) ; L_{2}\left(\mathscr{O} ; \mathbb{C}^{n}\right)\right), 0<T \leqslant \infty$, при некотором $1<p \leqslant \infty$. Тогда справедливо представление

$$
\mathbf{u}_{b, \varepsilon}(\cdot, t)=e^{-A_{b, \varepsilon} t} \boldsymbol{\phi}+\int_{0}^{t} e^{-A_{b, \varepsilon}(t-\tilde{t})} \mathbf{F}(\cdot, \tilde{t}) d \tilde{t} .
$$

Пусть $\mathbf{u}_{b, 0}(\mathbf{x}, t)$ - решение эффективной задачи

$$
\begin{cases}\frac{\partial \mathbf{u}_{b, 0}(\mathbf{x}, t)}{\partial t}=-b(\mathbf{D})^{*} g^{0} b(\mathbf{D}) \mathbf{u}_{b, 0}(\mathbf{x}, t)+\mathbf{F}(\mathbf{x}, t), & \mathbf{x} \in \mathscr{O}, 0<t<T, \\ \mathbf{u}_{b, 0}(\mathbf{x}, 0)=\boldsymbol{\phi}(\mathbf{x}), \quad \mathbf{x} \in \mathscr{O}, & 0<t<T .\end{cases}
$$

Аналогично $(12)$ имеем $\mathbf{u}_{b, 0}(\cdot, t)=e^{-A_{b}^{0} t} \boldsymbol{\phi}+\int_{0}^{t} e^{-A_{b}^{0}(t-\tilde{t})} \mathbf{F}(\cdot, \tilde{t}) d \tilde{t}$.

Отсюда и из (12) с помощью теоремы 1 и неравенства Гёльдера выводим следующий результат.

Теорема 5. Найдется число $\varepsilon_{0} \in(0,1]$, зависящее лищь от области $\mathscr{O} u$ решетки $\Gamma$, такое, что при $0<\varepsilon \leqslant \varepsilon_{0}$ u $0<t<T$ справедлива оценка

$$
\begin{aligned}
& \left\|\mathbf{u}_{b, \varepsilon}(\cdot, t)-\mathbf{u}_{b, 0}(\cdot, t)\right\|_{L_{2}(\mathscr{O})} \\
& \quad \leqslant C_{1}^{b} e^{-c_{b} t} \varepsilon\left(t+\varepsilon^{2}\right)^{-1 / 2}\|\phi\|_{L_{2}(\mathscr{O})}+\mathscr{C}_{1}^{b}(p) \theta_{p}(\varepsilon)\|\mathbf{F}\|_{\mathscr{H}_{p}(t)}, \quad b=D, N,
\end{aligned}
$$

əəe

$$
\theta_{p}(\varepsilon):= \begin{cases}\varepsilon^{2-2 / p}, & 1<p<2 \\ \varepsilon(|\ln \varepsilon|+1)^{1 / 2}, & p=2 \\ \varepsilon, & 2<p \leqslant \infty\end{cases}
$$

Аппроксимация решений в $H^{1}\left(\mathscr{O} ; \mathbb{C}^{n}\right)$ получается с помощью теоремы 2 и предложения 3. При этом мы предполагаем, что $p>2$. Осложнение возникает при аппроксимации интегрального члена в (12). Считая, что $t \geqslant \varepsilon^{2}$, мы разбиваем интеграл в (12) в сумму двух - по промежуткам $\left(0, t-\varepsilon^{2}\right)$ и $\left(t-\varepsilon^{2}, t\right)$; для первого применяем (6) или (8), а для второго - оценку (10). 
Введем обозначение $\mathbf{w}_{b, \varepsilon}(\cdot, t)=e^{-A_{b}^{0} \varepsilon^{2}} \mathbf{u}_{b, 0}\left(\cdot, t-\varepsilon^{2}\right)$, где $\mathbf{u}_{b, 0}-$ решение задачи (13). Тогда $\mathbf{w}_{b, \varepsilon}(\cdot, t)=e^{-A_{b}^{0} t} \phi+\int_{0}^{t-\varepsilon^{2}} e^{-A_{b}^{0}(t-\tilde{t})} \mathbf{F}(\cdot, \tilde{t}) d \tilde{t}$. Следующая теорема дает $H^{1}$-аппроксимацию решения $\mathbf{u}_{b, \varepsilon}(\cdot, t)$ и $L_{2}$-аппроксимацию потока $\mathbf{p}_{b, \varepsilon}(\cdot, t):=g^{\varepsilon} b(\mathbf{D}) \mathbf{u}_{b, \varepsilon}(\cdot, t)$.

Теорема 6. Пусть $p>2$. Найдется число $\varepsilon_{0} \in(0,1]$, зависящее лишь от области $\mathscr{O}$ и решетки $\Gamma$, такое, что при $b=D, N$ имеют место следуюшие утверждения.

$1^{\circ}$. Положим $\mathbf{v}_{D, \varepsilon}:=\Lambda^{\varepsilon} S_{\varepsilon} b(\mathbf{D}) P_{\mathscr{O}} \mathbf{w}_{D, \varepsilon}, \mathbf{v}_{N, \varepsilon}:=\Lambda^{\varepsilon} S_{\varepsilon} b(\mathbf{D}) P_{\mathscr{O}}\left(I-\mathscr{P}_{Z}\right) \mathbf{w}_{N, \varepsilon}$, $\mathbf{q}_{D, \varepsilon}:=\tilde{g}^{\varepsilon} S_{\varepsilon} b(\mathbf{D}) P_{\mathscr{O}} \mathbf{w}_{D, \varepsilon}, \mathbf{q}_{N, \varepsilon}:=\tilde{g}^{\varepsilon} S_{\varepsilon} b(\mathbf{D}) P_{\mathscr{O}}\left(I-\mathscr{P}_{Z}\right) \mathbf{w}_{N, \varepsilon}$. Прu $0<\varepsilon \leqslant \varepsilon_{0} u$ $\varepsilon^{2} \leqslant t<T$ справедливы оченки

$$
\begin{aligned}
& \left\|\mathbf{u}_{b, \varepsilon}(\cdot, t)-\mathbf{u}_{b, 0}(\cdot, t)-\varepsilon \mathbf{v}_{b, \varepsilon}(\cdot, t)\right\|_{H^{1}(\mathscr{O})} \\
& \leqslant 2 C_{2}^{b} e^{-c_{b} t} \varepsilon^{1 / 2} t^{-3 / 4}\|\phi\|_{L_{2}(\mathscr{O})}+\mathscr{C}_{2}^{b}(p) \rho_{p}(\varepsilon)\|\mathbf{F}\|_{\mathscr{H}_{p}(t)}, \\
& \left\|\mathbf{p}_{b, \varepsilon}(\cdot, t)-\mathbf{q}_{b, \varepsilon}(\cdot, t)\right\|_{L_{2}(\mathscr{O})} \\
& \leqslant 2 C_{3}^{b} e^{-c_{b} t} \varepsilon^{1 / 2} t^{-3 / 4}\|\phi\|_{L_{2}(\mathscr{O})}+\mathscr{C}_{3}^{b}(p) \rho_{p}(\varepsilon)\|\mathbf{F}\|_{\mathscr{H}_{p}(t)}, \\
& \rho_{p}(\varepsilon):= \begin{cases}\varepsilon^{1-2 / p}, & 2<p<4, \\
\varepsilon^{1 / 2}(|\ln \varepsilon|+1)^{3 / 4}, & p=4, \\
\varepsilon^{1 / 2}, & 4<p \leqslant \infty .\end{cases}
\end{aligned}
$$

$2^{\circ}$. Пусть $\Lambda \in L_{\infty}$. При $0<\varepsilon \leqslant \varepsilon_{0} u \varepsilon^{2} \leqslant t<T$ справедливы оченки

$$
\begin{aligned}
& \left\|\mathbf{u}_{b, \varepsilon}(\cdot, t)-\mathbf{u}_{b, 0}(\cdot, t)-\varepsilon \Lambda^{\varepsilon} b(\mathbf{D}) \mathbf{w}_{b, \varepsilon}(\cdot, t)\right\|_{H^{1}(\mathscr{O})} \\
& \quad \leqslant 2 \widetilde{C}_{2}^{b} e^{-c_{b} t} \varepsilon^{1 / 2} t^{-3 / 4}\|\phi\|_{L_{2}(\mathscr{O})}+\widetilde{\mathscr{C}}_{2}^{b}(p) \rho_{p}(\varepsilon)\|\mathbf{F}\|_{\mathscr{H}_{p}(t)}, \\
& \left\|\mathbf{p}_{b, \varepsilon}(\cdot, t)-\tilde{g}^{\varepsilon} b(\mathbf{D}) \mathbf{w}_{b, \varepsilon}(\cdot, t)\right\|_{L_{2}(\mathscr{O})} \\
& \quad \leqslant 2 \widetilde{C}_{3}^{b} e^{-c_{b} t} \varepsilon^{1 / 2} t^{-3 / 4}\|\boldsymbol{\phi}\|_{L_{2}(\mathscr{O})}+\widetilde{\mathscr{C}}_{3}^{b}(p) \rho_{p}(\varepsilon)\|\mathbf{F}\|_{\mathscr{H}_{p}(t)} .
\end{aligned}
$$

Замечание 7. Постоянные $\mathscr{C}_{j}^{b}(p), \widetilde{\mathscr{C}}_{j}^{b}(p)$ из неравенств $(14)-(18)$ зависят от тех же величин, что и $C_{j}^{b}, \widetilde{C}_{j}^{b}$ соответственно (см. замечание 4 ), а также от $p$.

\section{ЛитерАтУРА}

[1] A. Bensoussan, J.-L. Lions, G. Papanicolaou, Asymptotic Analysis for Periodic Structures, Studies in Math. and Appl., vol. 5, North-Holland, Amsterdam-New York, 1978. [2] Н. С. Бахвалов, Г. П. Панасенко, Осреднение прочессов в периодических средах, Наука, М., 1984. [3] В. В. Жиков, С. М. Козлов, О. А. Олейник, Усреднение дифференциальных операторов, Наука, М., 1993. [4] Т. А. Суслина, Функц. анализ и его прил., 38:4 (2004), 86-90. [5] Т. A. Suslina, in: Nonlinear Equations and Spectral Theory, Amer. Math. Soc. Transl. (2), vol. 220, Amer. Math. Soc., Providence, RI, 2007, 201-233. [6] V. V. Zhikov, S. E. Pastukhova, Russ. J. Math. Phys., 13:2 (2006), 224-237. [7] T. A. Suslina, Math. Model. Nat. Phenom., 5:4 (2010), 390-447. [8] Е. С. Василевская, Алгебра и анализ, 21:1 (2009), 3-60. [9] Е. С. Василевская, Т. А. Суслина, Алгебра и анализ, 24:2 (2012), 1-103. [10] Ю. М. Мешкова, Алгебра и анализ, 25:6 (2013), 125-177. [11] М. А. Пахнин, Т. А. Суслина, Алгебра и анализ, 24:6 (2012), 139-177. [12] T. A. Suslina, Mathematika, 59:2 (2013), 463-476. [13] T. A. Suslina, SIAM J. Math. Anal., 45:6 (2013), 3453-3493. [14] Т. А. Суслина, Функц. анализ и его прил., 48:4 (2014), 88-94. [15] J. Nečas, Direct Methods in the Theory of Elliptic Equations, Springer 
Monographs in Math., Springer-Verlag, Heidelberg-Dordrecht-London-New York, 2012. [16] И. М. Стейн, Сингулярные интегралы и дифференциальные свойства функций, Мир, М., 1973.

Санкт-Петербургский государственный университет, Лаборатория им. П. Л. Чебышева

Поступила в редакцию e-mail: juliavmeshke@yandex.ru 7 февраля 2014 г.

Санкт-Петербургский государственный университет, физический факультет

e-mail: suslina@list.ru

УДК 517.958+621.372.8

\section{О вычислении волноводной матрицы рассеяния для системы Максвелла*}

(c) 2015. Б. А. ПЛАмЕНЕВСКИЙ, А. С. ПОРЕЦКИЙ, О. В. САРАФАНОВ

Постановка краевой задачи. Пусть $G$ - область в $\mathbb{R}^{3}$, совпадающая вне большого шара с объединением непересекающихся полуцилиндров $\Pi_{+}^{q}=$ $\left\{\left(y^{q}, t^{q}\right): y^{q} \in \Omega^{q}, t^{q}>0\right\}$, где $q=1, \ldots, \mathscr{T}<\infty$ и $\Omega^{q}$ - ограниченная область в $\mathbb{R}^{2}$; граница $\partial G$ предполагается гладкой. В области $G$ рассматривается система Максвелла

с краевыми условиями

$$
\begin{aligned}
i \operatorname{rot} u^{2}(x)-k u^{1}(x) & =0, \\
-i \operatorname{div} u^{2}(x) & =0, \\
-i \operatorname{rot} u^{1}(x)-k u^{2}(x) & =0, \\
i \operatorname{div} u^{1}(x) & =0
\end{aligned}
$$

$$
\nu(x) \times u^{1}(x)=0, \quad\left\langle u^{2}(x), \nu(x)\right\rangle=0, \quad x \in \partial G,
$$

где $\langle\cdot, \cdot\rangle$ - скалярное произведение в $\mathbb{C}^{3}, \cdot \times \cdot-$ векторное произведение в $\mathbb{R}^{3}$, а $\nu$ - единичный вектор внешней нормали к $\partial G$. Положим $U=\left(u^{1}, u^{2}\right)$ и систему (1), (2) запишем в виде $\mathscr{M}(k) U=0$.

Собственные функции непрерывного спектра. Если для числа $k$ существует решение $U=\left(u^{1}, u^{2}\right)$ задачи $(1),(2)$ с оценкой $U(x)=O(|x|)$ при $|x| \rightarrow \infty$, не принадлежащее $L_{2}(G)$, то говорят, что $k$ - точка непрерывного спектра, а $U$ - отвечающая числу $k$ собственная функция непрерывного спектра. Известно [2], что непрерывный спектр задачи (1), (2) имеет вид $\left\{k \in \mathbb{R}:|k| \geqslant \mu_{\mathfrak{M}}^{1 / 2}\right\} \cup\{0\} ;$ здесь $\mu_{\mathfrak{M}}-$ наименьшее из положительных собственных чисел задач Неймана для оператора Лапласа $-\Delta$ в областях $\Omega_{q}$, $q=1, \ldots, \mathscr{T}$. Точка $k=0$ формально принадлежит непрерывному спектру, однако матрица рассеяния в этой точке не вводится, поэтому мы исключим ее из рассмотрения. Число $k$ называется собственным числом задачи (1), (2), если существует решение этой задачи, лежащее в $L_{2}(G)$; такое решение называется

* Исследование выполнено при поддержке грантов СПбГУ №11.38.666.2013 и РФФИ-1201-00247a. 